\title{
Review of: "Simultaneous construction of axial and planar chirality by gold/TY-Phos-catalyzed asymmetric hydroarylation"
}

\author{
Xinfang $\mathrm{Xu}^{1}$ \\ 1 Sun Yat-Sen University
}

Potential competing interests: The author(s) declared that no potential competing interests exist.

Chiral molecules hold a special place in synthetic chemistry, medicinal chemistry, materials, and many other interdisciplinary fields. However, general asymmetric catalytic approaches provide compounds with only one type of chiral element, such as center, axial, planar, or helic chirality. In this work, Zhang and coworkers have reported a gold-catalyzed asymmetric hydroarylation of ortho-alkynylaryl ferrocenes for the simultaneous construction of axial and planar chirality in these generated PAHs. Although the utility is not very clear so far, the development of novel catalytic method for the construction of chiral entities with more than one type of chirality is interesting and appealing considering the diversified asymmetric elements in biomolecules and chiral recognition in a variety of bioactivity processes.

There are several concerns not clear with this study which include the following:

1. Installation of multi-stereochemical elements within a single step is intrinsically challenging and has been rarely reported. In this work, the author developed an effective strategy for the synthesis of PAHs with simultaneous construction of planar and axial chirality by an Au-catalysed asymmetric intermolecular hydroarylation with "homemade" chiral TY-Phos ligands on one step. These chiral PAHs are isolated in high to excellent yields with generally around $90 \%$ ee. Although the excellent diastereoselectivities could be obtained by increasing the steric hindrance of the substitution group on the ortho-position. Thus, further optimization could be expected by modification of the chiral ligand or catalytic conditions to enable high stereoselectivity with broad substrate scope.

2. This method is a unique and effective approach for the construction of chiral PAHs with axial and planar chirality. Moreover, the catalytic alkyne cyclization has been one of the practical reactions for the construction of aromatic rings. Thus, this work would inspiration the synthetic chemists to development novel catalytic methods for the synthesis chiral PAHs with divers combination of different chirality.

3. Due to the unique liner geometry of the key in gold-catalyzed alkyne functionalization, there are only very few limited examples of chiral gold complexes that have been disclosed to exhibit high enantioselectivity in corresponding catalytic alkyne transformations. Thus, this work not only shows additional example of asymmetric catalytic alkyne functionalization, but also demonstrated a novel gold complex that is a robust and effective catalyst, which could be potentially subjected to a variety of 
asymmetric transformations with the structure optimization.

4. In this work, the authors have also conducted the DFT calculation to rationalize the asymmetric introduction and stereo-determination steps. It's also very important to illustrate the chirality transfer process. For example, recent work by $\mathrm{Xu}$ and Yu has disclosed a transient-axial-chirality controlled "axial to point chirality transfer" process for the asymmetric alkyne functionalization (Nat. Commun. $2020,11,2363)$. These detailed mechanism study would be the primary task and very useful for the selective construction of chiral molecules that possess more than one chiral element. 